NASA/TM-2014-218175

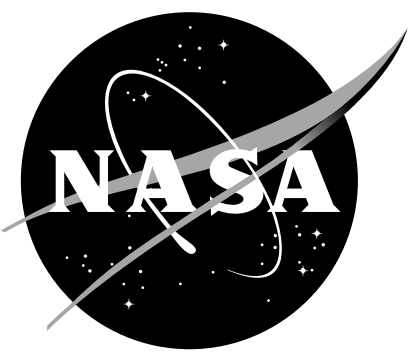

\title{
First-Order Hyperbolic System Method for Time-Dependent Advection-Diffusion Problems
}

Alireza Mazaheri

Langley Research Center, Hampton, Virginia

Hiroaki Nishikawa

National Institute of Aerospace, Hampton, Virginia 


\section{NASA STI Program . . . in Profile}

Since its founding, NASA has been dedicated to the advancement of aeronautics and space science. The NASA scientific and technical information (STI) program plays a key part in helping NASA maintain this important role.

The NASA STI program operates under the auspices of the Agency Chief Information Officer. It collects, organizes, provides for archiving, and disseminates NASA's STI. The NASA STI program provides access to the NASA Aeronautics and Space Database and its public interface, the NASA Technical Report Server, thus providing one of the largest collections of aeronautical and space science STI in the world. Results are published in both non-NASA channels and by NASA in the NASA STI Report Series, which includes the following report types:

- TECHNICAL PUBLICATION. Reports of completed research or a major significant phase of research that present the results of NASA Programs and include extensive data or theoretical analysis. Includes compilations of significant scientific and technical data and information deemed to be of continuing reference value. NASA counterpart of peerreviewed formal professional papers, but having less stringent limitations on manuscript length and extent of graphic presentations.

- TECHNICAL MEMORANDUM. Scientific and technical findings that are preliminary or of specialized interest, e.g., quick release reports, working papers, and bibliographies that contain minimal annotation. Does not contain extensive analysis.

- CONTRACTOR REPORT. Scientific and technical findings by NASA-sponsored contractors and grantees.
- CONFERENCE PUBLICATION. Collected papers from scientific and technical conferences, symposia, seminars, or other meetings sponsored or cosponsored by NASA.

- SPECIAL PUBLICATION. Scientific, technical, or historical information from NASA programs, projects, and missions, often concerned with subjects having substantial public interest.

- TECHNICAL TRANSLATION. English-language translations of foreign scientific and technical material pertinent to NASA's mission.

Specialized services also include organizing and publishing research results, distributing specialized research announcements and feeds, providing information desk and personal search support, and enabling data exchange services.

For more information about the NASA STI program, see the following:

- Access the NASA STI program home page

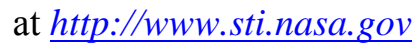

- E-mail your question to help@sti.nasa.gov

- Fax your question to the NASA STI Information Desk at 443-757-5803

- Phone the NASA STI Information Desk at 443-757-5802

- Write to:

STI Information Desk NASA Center for AeroSpace Information 7115 Standard Drive Hanover, MD 21076-1320 


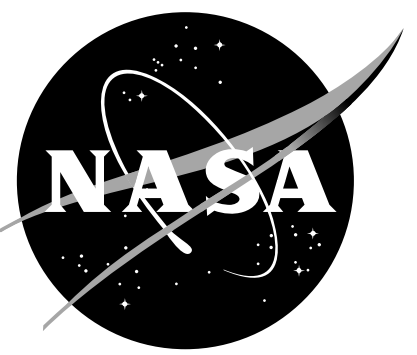

\section{First-Order Hyperbolic System Method for Time-Dependent Advection-Diffusion Problems}

Alireza Mazaheri

Langley Research Center, Hampton, Virginia

Hiroaki Nishikawa

National Institute of Aerospace, Hampton, Virginia

National Aeronautics and

Space Administration

Langley Research Center

Hampton, Virbinia 23681-2199 
The use of trademarks or names of manufacturers in this report is for accurate reporting and does not constitute an official endorsement, either expressed or implied, of such products or manufacturers by the National Aeronautics and Space Administration.

Available from:

NASA Center for AeroSpace Information

7115 Standard Drive

Hanover, MD 21076-1320

443-757-5802 


\section{Abstract}

A time-dependent extension of the first-order hyperbolic system method [J. Comput. Phys., 227 (2007) 315-352] for advection-diffusion problems is introduced. Diffusive/viscous terms are written and discretized as a hyperbolic system, which recovers the original equation in the steady state. The resulting scheme offers advantages over traditional schemes: a dramatic simplification in the discretization, high-order accuracy in the solution gradients, and orders-ofmagnitude convergence acceleration. The hyperbolic advection-diffusion system is discretized by the second-order upwind residual-distribution scheme in a unified manner, and the system of implicit-residual-equations is solved by Newton's method over every physical time step. The numerical results are presented for linear and nonlinear advection-diffusion problems, demonstrating solutions and gradients produced to the same order of accuracy, with rapid convergence over each physical time step, typically less than five Newton iterations. 


\section{Contents}

1 Introduction 3

2 Hyperbolic Advection-Diffusion System 4

3 Discretization, Steady Solver, and Boundary Condition $\quad 6$

3.1 Discretization . . . . . . . . . . . . 6

3.2 Steady Solver . . . . . . . . . . . . . . . . . . . 8

3.3 Boundary Condition . . . . . . . . . . . . . . . . . . . . 10

4 Extension to Time-Dependent Problems 11

5 Extension to Nonlinear Advection-Diffusion System 13

6 Results $\quad 17$

6.1 Steady Linear Advection-Diffusion (High Re Applications) . . 17

6.2 Unsteady Linear Advection-Diffusion . . . . . . . . . . . . . . 19

6.3 Unsteady Nonlinear Advection-Diffusion . . . . . . . . . . . . 23

6.4 Accuracy Verification . . . . . . . . . . . . 25

$\begin{array}{llr}7 & \text { Conclusions } & 28\end{array}$ 


\section{Introduction}

In this paper, we introduce an unsteady hyperbolic advection-diffusion scheme. The first-order hyperbolic system method was initially proposed in Ref. [1] as a radical approach to steady diffusion problems: discretize an equivalent first-order hyperbolic system to solve the diffusion equation. It has a number of attractive features such as the discretization of high-order derivatives by methods for hyperbolic systems, equal order of accuracy for the solution and the gradients (viscous/heat fluxes), and orders of magnitude acceleration in steady convergence. These advantages have been demonstrated for the diffusion equation [1] and the advection-diffusion equation [2] by the second-order residual-distribution method, and for the compressible Navier-Stokes equations [3] by the second-order finite-volume method, and for the advectiondiffusion equation by first, second and third order finite-volume methods $[4,5]$. These lines of development, however, have been restricted exclusively to steady state problems. Towards enabling accurate practical time-dependent computations by the first-order hyperbolic system method, this paper presents the first study on the unsteady extension of the first-order hyperbolic system method. We demonstrate that time-accurate schemes constructed via the hyperbolic method produce high-order accurate solutions and derivatives at every time step and allow the construction of a highly efficient inner solver for implicit time stepping.

Time-dependent computations are possible by implicit time-integration methods such as the second-order backward-difference scheme, which is widely used for practical computations. To perform the implicit time integration, it is required to solve a system of global time-dependent residual equations over each time step. We employ a steady solver developed by the first-order hyperbolic system method to solve the system efficiently and accurately. The spatial discretization is performed by the residual-distribution method as in Refs. [1,2]. Taking advantage of the compactness of the residual-distribution schemes, we develop a fully-implicit steady solver with exact linearization, i.e., Newton's method, for a second-order upwind scheme. The strategy taken here may be thought of as a dual-time formulation [6]. However, we introduce a pseudo-time just for convenience of discretization: the first-order system is hyperbolic in the pseudo-time and thus will be discretized by the upwind residual-distribution scheme. Once the spatial discretization is complete, the pseudo-time step is taken to be infinity to define the system of time-dependent residual equations, which is then solved by Newton's method. In this paper, we consider one-dimensional linear and nonlinear advection-diffusion equations. These examples are sufficient to illustrate the general methodology for enabling time-dependent computations by the hyperbolic method; this is the main goal of this paper. The introduction of this unsteady hyperbolic advection-diffusion scheme along with its great advantages is served as a foun- 
dation for the extension of the scheme to higher-dimensions and more complex systems.

It is emphasized also that there are real practical applications that onedimensional analyses are sufficient and routinely being used in industries (e.g., NASA). For instance, in-depth material thermal response calculations of thermal protection systems of atmospheric entry vehicles, where advection-diffusion equation is solved through the material, is a prime example of such applications [7-9]. Another example is the experimental aeroheating data reduction that are obtained with global phosphor thermography techniques [10,11]. Efficient and accurate one-dimensional schemes as presented in this paper will significantly improve these analyses and could potentially make such calculations part of the routine aerothermodynamic environment predictions. The present paper serves, therefore, also as an important foundation for the extension to more practical one-dimensional problems.

There exist other methods for enabling time-accurate computations by the residual-distribution method: the Crank-Nicolson method [12], the spacetime method [13-15], the backward-difference method [16,17], and the explicit Runge-Kutta-type method [18]. These methods are equally applicable to the construction of time-accurate schemes in the hyperbolic method. In this paper, we employ the backward-difference method for simplicity but without losing practical applicability. The main difference between our time-integration method and those in Refs. [16,17] lies in the method for solving the system of residual equations arising from the implicit time integration scheme: we employ Newton's method whereas Ref. [16] employs the dual-time stepping method with a pseudo-time marching iteration and Ref. [17] employs the multigrid method with a point-implicit iteration.

The paper is organized as follows. In the next section, the hyperbolic advection-diffusion system is described. In Section 3, a compact second-order residual-distribution scheme, a steady solver, and the boundary conditions are discussed. In Section 4, the construction of time-accurate schemes is given. In Section 5, the time-accurate scheme is extended to a nonlinear advectiondiffusion equation. In Section 6, numerical results are presented. Finally, Section 7 concludes the study with remarks.

\section{Hyperbolic Advection-Diffusion System}

Consider the one-dimensional (1-D) advection-diffusion equation,

$$
\partial_{t} u+a \partial_{x} u=\nu \partial_{x x} u
$$

where $a$ and $\nu$ are both taken to be positive constant. We will follow the procedure described in Ref. [2] and rewrite the above equation with a first- 
order advection-diffusion system:

$$
\begin{aligned}
\partial_{t} u & =-a \partial_{x} u+\nu \partial_{x} p \\
\partial_{t} p & =\left(\partial_{x} u-p\right) / T_{r}
\end{aligned}
$$

where the relaxation time, $T_{r}>0$, is arbitrary. Towards the steady state, the variable $p$ approaches the solution gradient and hence the above equation becomes identical to the original advection-diffusion equation in the steady state. In the vector form, the first-order advection-diffusion system can be written as

$$
\partial_{t} \mathbf{U}+\mathbf{A} \partial_{x} \mathbf{U}=\mathbf{S}
$$

where

$$
\mathbf{U}=\left[\begin{array}{l}
u \\
p
\end{array}\right], \quad \mathbf{A}=\left[\begin{array}{cc}
a & -\nu \\
-1 / T_{r} & 0
\end{array}\right], \quad \mathbf{S}=\left[\begin{array}{c}
0 \\
-p / T_{r}
\end{array}\right]
$$

The above system is hyperbolic in time because it has the following real eigenvalues for any positive $T_{r}$ :

$$
\lambda_{1}=\frac{a}{2}\left[1-\sqrt{1+\frac{4 \nu}{a^{2} T_{r}}}\right], \quad \lambda_{2}=\frac{a}{2}\left[1+\sqrt{1+\frac{4 \nu}{a^{2} T_{r}}}\right],
$$

with linearly independent eigenvectors [1].

Note that the above hyperbolic formulation is equivalent to the original Eq. (1) only in the steady state. The time derivatives in the hyperbolic system, at least $\partial_{t} p$, should therefore be taken as pseudo-time derivatives. They serve mainly as a guide for discretization by making the whole system hyperbolic in time for which various discretization techniques are available, e.g., upwinding. The benefits are, however, much more than just the convenience in discretization. First, the hyperbolic discretization typically results in a strong coupling between the two variables and achieves the equal order of accuracy for $u$ and $p$ on arbitrary grids. Second, the resulting explicit numerical scheme is stable with an $O(h)$ time step through the diffusion limit or $O(1 / h)$ condition number in the residual Jacobian for implicit solvers. It has been shown to yield $O(1 / h)$ acceleration in convergence over traditional methods for the diffusion equation $[1,4]$, for the advection-diffusion equation $[2,5]$, and for the compressible Navier-Stokes equations [3]. Unsteady computations are possible by implicit time stepping, which is the main subject of this paper.

In the next section, we first fully describe the advection-diffusion equation discretization process, the implicit steady state formulation, and the boundary condition implementation in the steady state limit. We then extend the scheme to time-accurate and nonlinear advection-diffusion equation in the sections that follow. 


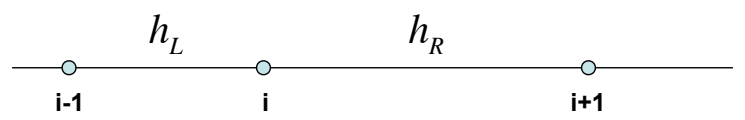

Figure 1: Schematic of grid spacing for a 1-D grid.

\section{Discretization, Steady Solver, and Bound- ary Condition}

\subsection{Discretization}

To discretize the first-order hyperbolic advection-diffusion system, we employ Residual-Distribution (RD) method. The method consists of two steps; 1) residual evaluation over the cells (or elements), and 2) distribution of the residuals to the nodes.

Consider a one-dimensional domain discretized with $N$ nodes that are distributed arbitrarily over the domain of interest with the solution, $u$, and the solution gradient, $p$, data stored at the nodes denoted by $x_{i}, i=1,2,3, \ldots, N$ (Fig.1). We define the cell-residual $\boldsymbol{\Phi}^{\mathbf{C}}$ by integrating the spatial part of the Eq. (4) over the cell, $C$, defined by the nodes $i$ and $i+1$ :

$$
\boldsymbol{\Phi}^{\mathbf{C}}=\int_{\mathbf{x}_{\mathbf{i}}}^{\mathbf{x}_{\mathbf{i}+\mathbf{1}}}\left(-\mathbf{A} \partial_{\mathbf{x}} \mathbf{U}+\mathbf{S}\right) \mathbf{d} \mathbf{x}=\left\{\begin{array}{l}
-a\left(u_{i+1}-u_{i}\right)+\nu\left(p_{i+1}-p_{i}\right) \\
\frac{1}{T_{r}}\left[u_{i+1}-u_{i}-\frac{p_{i+1}+p_{i}}{2}\left(x_{i+1}-x_{i}\right)\right]
\end{array}\right.
$$

Note that the source term integration has been evaluated by the trapezoidal rule to ensure second-order accuracy. It should be noted also that the flux balance terms e.g., $u_{x}$, has been integrated exactly, which is not possible in higher dimensions and a high-order quadrature would be required to achieve the equal order of accuracy for the solution and the gradients in the residual-distribution method as described in Ref. [2]. We again remark that the relaxation time, $T_{r}$, is arbitrary because in the steady state limit $p=u_{x}$ for any $T_{r}$. We complete the evaluation of the cell residual with the definition of $T_{r}$. Using the dimensional analysis [2], this is defined as

$$
T_{r}=\frac{L_{r}}{\max \left(\lambda_{1}, \lambda_{2}\right)}=\frac{L_{r}}{\frac{a}{2}\left[1+\sqrt{1+\frac{4 \nu}{a^{2} T_{r}}}\right]},
$$

where $L_{r}$ is a length scale that may be optimized using Fourier analysis to enhance the convergence [19]. The effect of the optimized length scale remains to be investigated. Here we use the value recommended in Ref. $[1,4,5]$ :

$$
L_{r}=\frac{1}{2 \pi} .
$$


We now solve for $T_{r}$ from Eq. (8):

$$
T_{r}=\frac{L_{r}}{a+\nu / L_{r}} .
$$

Substituting back the relaxation time into Eq. (6), we express the eigenvalues as

$$
\lambda_{1}=-\nu / L_{r}, \lambda_{2}=a+\nu / L_{r} .
$$

At this point the residual evaluation is completed. We now define the distribution of the residuals. We achieve this by first diagonalizing the matrix A with the following right and left eigenvectors:

$$
R=\left[\begin{array}{cc}
-\lambda_{1} T_{r} & -\lambda_{2} T_{r} \\
1 & 1
\end{array}\right], \quad L=\frac{a L_{r}+\nu}{L_{r}\left(a L_{r}+2 \nu\right)}\left[\begin{array}{cc}
1 & -L_{r} \\
-1 & \left(\nu L_{r}\right) /\left(a L_{r}+\nu\right)
\end{array}\right]
$$

We then expand the matrix $\mathbf{A}$ to two separate parts that are distinguished by its corresponding wave speeds (eigenvalues):

$$
A=R \Lambda L=\lambda_{1} B^{-}+\lambda_{2} B^{+},
$$

where

$$
\begin{gathered}
\Lambda=\left[\begin{array}{cc}
\lambda_{1} & 0 \\
0 & \lambda_{2}
\end{array}\right], \\
B^{-}=\frac{1}{a L_{r}+2 \nu}\left[\begin{array}{cc}
\nu & \nu L_{r} \\
\left(a L_{r}+\nu\right) / L_{r} & a L_{r}+\nu
\end{array}\right], \\
B^{+}=\frac{1}{a L_{r}+2 \nu}\left[\begin{array}{cc}
a L_{r}+\nu & -\nu L_{r} \\
-\left(a L_{r}+\nu\right) / L_{r} & \nu
\end{array}\right] .
\end{gathered}
$$

The matrices $B^{+}$and $B^{-}$project the solution, respectively, onto the leftand right-running waves. Hence, this is upwinding. We also note that the distribution matrices have the following property

$$
B^{-}+B^{+}=I,
$$

which is required for conservation. We can now distribute the cell residual $\Phi^{\mathrm{C}}$ to the nodes using the projection matrices $B^{+}$and $B^{-}$as described in Fig. 2. The distribution is done this way because the left running wave, $\lambda_{2}$, for example, is contributing to the $B^{+}$and thus the cell residual on the left cell is weighted with the $B^{+}$. After the distribution step, we obtain the following semi-discrete scheme in pseudo-time:

$$
\frac{d \mathbf{U}_{i}}{d \tau}=\frac{1}{h_{i}}\left(B^{+} \Phi^{L}+B^{-} \Phi^{R}\right),
$$

where $\Phi^{L}$ and $\Phi^{R}$ denote the cell-residuals over the left and right cells of the node $i$, respectively, and $h_{i}$ is the dual volume (see Fig. 1) defined by

$$
h_{i}=\frac{h_{L}+h_{R}}{2}, \quad h_{L}=x_{i}-x_{i-1}, \quad h_{R}=x_{i+1}-x_{i} .
$$




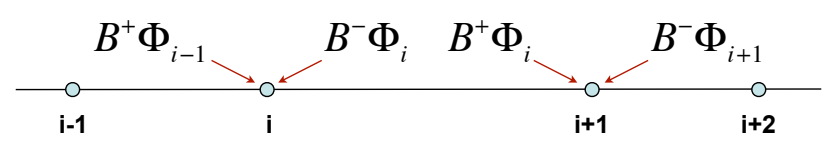

Figure 2: Residual distribution to the nodes.

\subsection{Steady Solver}

Equation (18) can be solved with either an explicit or an implicit formulation. We choose to focus on the implicit formulation for a better transition to the next sections, where we extend this scheme to general nonlinear timedependent advection-diffusion problems. Therefore, we drop the pseudo-time derivative from (18) and solve the resulting system of steady residual equations,

$$
0=\text { Res }
$$

by a fully implicit solver. We remark that in some complex problems the pseudo time derivative should be kept for stability purposes. In our onedimensional problems, however, we could choose an infinite pseudo time step without encountering any stability issue.

The implicit formulation is defined by

$$
\mathbf{U}^{k+1}=\mathbf{U}^{k}+\Delta \mathbf{U}^{k}
$$

where $\mathbf{U}=\left(u_{1}, p_{1}, u_{2}, p_{2}, \ldots, u_{N}, p_{N}\right)$ and $k$ is the iteration counter. The correction $\Delta \mathbf{U}^{k}=\mathbf{U}^{k+1}-\mathbf{U}^{k}$ is determined as the solution to the linear system:

$$
\frac{\partial \boldsymbol{R e s}}{\partial \mathbf{U}} \Delta \mathbf{U}^{k}=-\boldsymbol{R e s}^{k}
$$

where $\boldsymbol{R e s}^{k}$ is the steady residual vector evaluated by $\mathbf{U}^{k}$. The Jacobian matrix is sparse, having three $2 \times 2$ blocks in each row for all interior nodes and two blocks for boundary nodes. The $i$-th interior pair of row of the linear system is given by

$$
J_{i-1} \Delta U_{i-1}^{k}+J_{i} \Delta U_{i}^{k}+J_{i+1} \Delta U_{i+1}^{k}=-\frac{1}{h_{i}}\left(B^{+} \Phi^{L}+B^{-} \Phi^{R}\right)^{k},
$$


where $\Delta U_{i-1}^{k}=\left(\Delta u_{i-1}^{k}, \Delta p_{i-1}^{k}\right), \Delta U_{i}^{k}=\left(\Delta u_{i}^{k}, \Delta p_{i}^{k}\right), \Delta U_{i+1}^{k}=\left(\Delta u_{i+1}^{k}, \Delta p_{i+1}^{k}\right)$,

$$
\begin{aligned}
J_{i-1} & =\frac{1}{h_{i}} B^{+} \frac{\partial \Phi^{L}}{\partial U_{i-1}} \\
J_{i} & =\frac{1}{h_{i}}\left(B^{+} \frac{\partial \Phi^{L}}{\partial U_{i}}+B^{-} \frac{\partial \Phi^{R}}{\partial U_{i}}\right) \\
J_{i+1} & =\frac{1}{h_{i}} B^{-} \frac{\partial \Phi^{R}}{\partial U_{i+1}}
\end{aligned}
$$

and the derivatives of the cell-residuals are given by

$$
\begin{gathered}
\frac{\partial \Phi^{R}}{\partial U_{i}}=\left[\begin{array}{cc}
a & -\nu \\
-\frac{1}{T_{r}} & -\frac{h_{R}}{2 T_{r}}
\end{array}\right], \\
\frac{\partial \Phi^{L}}{\partial U_{i}}=\left[\begin{array}{cc}
-a & \nu \\
\frac{1}{T_{r}} & -\frac{h_{L}}{2 T_{r}}
\end{array}\right] .
\end{gathered}
$$

The linear system may be solved efficiently by Thomas' algorithm, which is an $O(N)$ method. In this paper, however, we employ the Gauss-Seidel (GS) relaxation, which is also an $O(N)$ method for the discretization arising from hyperbolic advection-diffusion system and extends straightforwardly to more complex systems, irregular grids, and higher dimensions, whereas Thomas' algorithm is not applicable in higher dimensions. The GS relaxation scheme may be applied equation by equation (decoupled relaxation) or node by node (collective relaxation) updating the solution set at a node simultaneouously [20]. In this work, the collective GS relaxation is employed for robustness; the decoupled GS relaxation was found to give better convergence in most cases but also found to be unstable in some cases whereas the collective relaxation encountered no such problems. In actual computations, we do not fully solve but relax the linear system to reduce the linear residual by two or three orders of magnitude.

Note that the Jacobian is exact and thus the implicit algorithm is Newton's method. It is one of the advantages of the residual-distribution method that second-order accuracy is achieved within a compact stencil, thus allowing the exact linearization with a sparse Jacobian matrix. The same is true for extension to higher dimensions. For linear problems, the method is essentially a direct method. That is, if we solve the linear system exactly, then we get the solution at the first Newton iteration. 

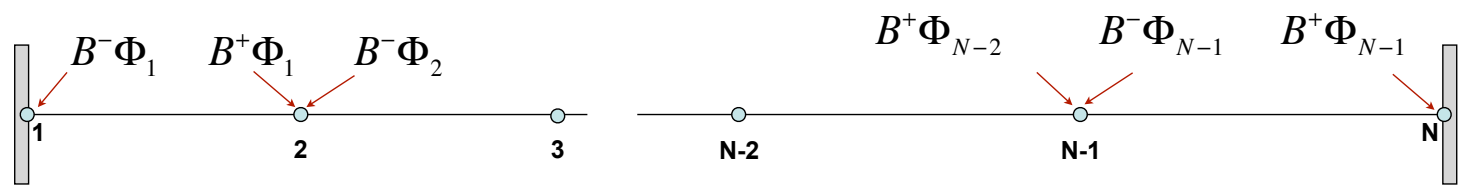

Figure 3: Schematic of the Dirichlet and the Neumann boundary conditions implementation in the hyperbolic advection-diffusion scheme.

\subsection{Boundary Condition}

In this section we describe the boundary condition implementation for our firstorder hyperbolic system scheme. Because in our hyperbolic advection-diffusion system the second equation in the hyperbolic system solves for the solution gradient, $p$, the Neumann boundary condition is implemented exactly in the same way as the Dirichlet boundary condition. Therefore, we only show the formulation for the $u$ variable and the same can be repeated for the $p$ variable. Note that the discrete problem has a unique solution for linear problems with two values given at the left and right boundaries (this is consistent with the differential equation allowing two boundary conditions): $2(N-1)$ cell-residuals for $2 N-2$ unknowns, which is the case in both of the following boundary conditions.

\section{Dirichlet or Neumann Boundary Condition}

For Dirichlet or Neumann boundary condition type schematically shown in Fig. 3, the discretized equation for the boundary condition imposed on $u$ takes the following form at the first node:

$$
J_{1}^{*} \Delta U_{1}^{k}+J_{2}^{*} \Delta U_{2}^{k}=-\frac{1}{h_{1}}\left(B^{-} \Phi^{R}\right)^{k},
$$

where for the imposed boundary condition on the $u$ variable, the Jacobian matrices are

$$
\begin{aligned}
& J_{1}^{*}=\frac{1}{h_{1}} B^{-}\left[\begin{array}{cc}
1 & 0 \\
\frac{\partial \Phi^{R}(2)}{\partial u_{1}} & \frac{\partial \Phi^{R}(2)}{\partial p_{1}}
\end{array}\right], \\
& J_{2}^{*}=\frac{1}{h_{2}} B^{-}\left[\begin{array}{cc}
0 & 0 \\
\frac{\partial \Phi^{R}(2)}{\partial u_{2}} & \frac{\partial \Phi^{R}(2)}{\partial p_{2}}
\end{array}\right],
\end{aligned}
$$

where $\Phi^{R}(2)$ denotes the second component of $\Phi^{R}$ corresponding to the second equation (the $p$ equation) of the first-order hyperbolic system. The same process is repeated for imposed boundary condition on the last node (for either $u$ or $p$.) 


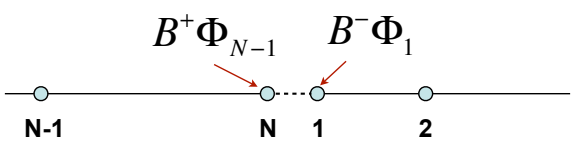

Figure 4: Schematic of the periodic boundary condition implementation in the hyperbolic advection-diffusion scheme.

\section{Periodic Boundary Condition}

To implement a periodic boundary condition we set $\mathbf{U}_{N}=\mathbf{U}_{1}$ in Eq. (23) and obtain the following discretized equation and applied it on both two boundaries (Fig. 4):

$$
J_{N-1} \Delta U_{N-1}^{k}+J_{1} \Delta U_{1}^{k}+J_{2} \Delta U_{2}^{k}=-\frac{\left(B^{+} \Phi^{L}+B^{-} \Phi^{R}\right)^{k}}{h_{1}},
$$

where $L$ denotes the cell defined by the nodes $N-1$ and $N$, and $R$ denotes the cell defined by the nodes 1 and 2 .

\section{Extension to Time-Dependent Problems}

We extend the steady state formulation by modifying the first-order hyperbolic system given by Eq. (2) and rewriting it as a dual-time step system:

$$
\begin{aligned}
\partial_{\tau} u & =-a u_{x}+\nu \partial_{x} p-\frac{\alpha}{\Delta t} u+s, \\
\partial_{\tau} p & =\left(\partial_{x} u-p\right) / T_{r},
\end{aligned}
$$

where $t$ is the physical time, $\Delta t$ is the physical time step, $\tau$ is the pseudo-time, and $s$ and $\alpha$ both depend on the physical time-step discretization scheme. Adopting first- or second-order Backward-Differencing-Formulation (BDF1 or BDF2, respectively) for the physical time integration, the $\alpha$ and $s(x)$ terms are:

$$
\begin{array}{lll}
\alpha=1, & s=\frac{u^{n}}{\Delta t} & : B D F 1, \\
\alpha=\frac{2 \Delta t^{n-1}+\Delta t^{n}}{\Delta t^{n-1}+\Delta t^{n}}, & s=\frac{\Delta t^{n-1}+\Delta t^{n}}{\Delta t^{n-1} \Delta t^{n}} u^{n}-\frac{\Delta t^{n-1}}{\Delta t^{n}\left(\Delta t^{n-1}+\Delta t^{n}\right)} u^{n-1} & : B D F 2,
\end{array}
$$

where a nonuniform time step formulation is used in the BDF2 formulation. The BDF1 is only used at the very beginning of the simulation with an initial time interval of $\Delta t^{0}\left(<<\Delta t^{n}\right)$. After the first time interval, BDF2 with a uniform time step, $\Delta t$, is used. This procedure will ensure a second-order 
accurate result through all times. The use of BDF1 is necessary because explicit time stepping is not possible with the hyperbolic system method.

We solve the above system over each physical time interval to obtain the steady state solution in the pseudo-time space. In the steady state in $\tau$, we have $p=\partial_{x} u$ and therefore recover the consistent implicit time discretization scheme for the original time-dependent advection-diffusion equation.

In the dual-time step system, the physical time derivative term acts as a source term to the first equation. This system thus shares the same eigenvalues and eigenvectors as the steady hyperbolic advection-diffusion system. We will see this more clearly by writing the time-dependent hyperbolic system in the vector form:

$$
\frac{\partial \mathbf{U}}{\partial \tau}+\mathbf{A} \frac{\partial \mathbf{U}}{\partial x}=\mathbf{D U}+\mathbf{S}
$$

where

$$
\mathbf{A}=\left[\begin{array}{cc}
a & -\nu \\
-1 / T_{r} & 0
\end{array}\right], \quad \mathbf{D}=\left[\begin{array}{cc}
-\alpha / \Delta t & 0 \\
0 & -1 / T_{r}
\end{array}\right], \quad \mathbf{S}=\left[\begin{array}{c}
s(x) \\
0
\end{array}\right]
$$

Because of the similarity in wave definitions in both time-dependent and steady hyperbolic systems, the distribution matrices $B^{-}$and $B^{+}$are the same for both of these systems. Once the discretization is obtained, we drop the pseudo-time derivatives and solve the resulting system of discrete equations by Newton's method as described in Section 3.2. To develop a time-dependent scheme, we therefore only need to add the source term effect in the cell-residual.

The cell-residual $\mathbf{\Phi}^{\mathbf{C}}$ of the time-dependent hyperbolic advection-diffusion system is

$$
\begin{aligned}
\Phi^{C} & =\int_{x_{i}}^{x_{i+1}}\left(-\mathbf{A} \mathbf{U}_{x}+\mathbf{D} \mathbf{U}+\mathbf{S}\right) d x \\
& =\left[\begin{array}{r}
-a\left(u_{i+1}-u_{i}\right)+\nu\left(p_{i+1}-p_{i}\right)-\left(x_{i+1}-x_{i}\right) \frac{\alpha}{\Delta t}\left(u_{i+1}+u_{i}\right) / 2 \\
\frac{1}{T_{r}}\left[u_{i+1}-u_{i}-\frac{p_{i+1}+p_{i}}{2}\left(x_{i+1}-x_{i}\right)\right]
\end{array}\right] \\
+ & {\left[\begin{array}{c}
\left(x_{i+1}-x_{i}\right)\left(s_{i+1}+s_{i}\right) / 2 \\
0
\end{array}\right]^{n-1, n} }
\end{aligned}
$$

where $k$ and $n$ are the Newton iteration counter and the physical time index, respectively. Note that the second term of the Eq. (38), which is computed at the two previous physical time steps, is constant during the Newton iteration, and thus will not contribute to the Jacobian. The residual Jacobians needed in the Newton solver are exactly in the same form as Eq. (26), but the derivatives of the cell-residuals now include the contribution from the physical time 
derivative:

$$
\begin{gathered}
\frac{\partial \Phi_{R}}{\partial U_{i}}=\left[\begin{array}{cc}
a-h_{R} \frac{\alpha}{2 \Delta t} & -\nu \\
-\frac{1}{T_{r}} & -\frac{h_{R}}{2 T_{r}}
\end{array}\right], \\
\frac{\partial \Phi_{L}}{\partial U_{i}}=\left[\begin{array}{cc}
-a-h_{L} \frac{\alpha}{2 \Delta t} & \nu \\
\frac{1}{T_{r}} & -\frac{h_{L}}{2 T_{r}}
\end{array}\right] .
\end{gathered}
$$

The hyperbolic advection-diffusion scheme is now time-accurate. At each physical time level $n$, we solve the pseudo steady problem by Newton's method with the current solution at $n$ as the initial solution.

\section{Extension to Nonlinear Advection-Diffusion System}

Consider the generalized time-dependent nonlinear advection-diffusion equation:

$$
\partial_{t} u+\partial_{x} f=\partial_{x}\left(\nu u_{x}\right)
$$

where $f$ is a nonlinear function of $u$ and $\nu=\nu(u)$ is a function of $u$. We write the above equation using the dual-time stepping formulation in the following first-order hyperbolic system as

$$
\begin{aligned}
\partial_{\tau} u & =-\partial_{x} f+\partial_{x} p-\frac{\alpha}{\Delta t} u+s, \\
\frac{T_{r}}{\nu(u)} \partial_{\tau} p & =\partial_{x} u-p / \nu(u) .
\end{aligned}
$$

This is a preconditioned conservative form introduced in Ref. [3] to extend the hyperbolic method to nonlinear equations. Note that the variable $p$ will be the diffusive flux in the pseudo steady state, not the gradient. In the vector form, the preconditioned conservative system is written as

$$
\mathbf{P}^{-1} \mathbf{U}_{\tau}=-\mathbf{F}_{\mathbf{x}}+\mathbf{S}
$$

where

$$
\mathbf{P}^{-1}=\left(\begin{array}{cc}
1 & 0 \\
0 & T_{r} / \nu(u)
\end{array}\right), \mathbf{F}=\left(\begin{array}{c}
f-p \\
-u
\end{array}\right), \mathbf{S}=\left(\begin{array}{c}
\frac{-\alpha}{\Delta t} u+s(x) \\
-p / \nu(u)
\end{array}\right)
$$

and $\mathbf{P}$ is the preconditioning matrix. The main role of the preconditioning matrix here is not to optimize the condition number of the differential system, but rather to simplify the discretization. In particular, in this form, there is 
no need to differentiate the diffusion coefficient with respect to the solution to derive the flux Jacobian. Multiplying both sides by $\mathbf{P}$ from the left, the flux term becomes

$$
\mathbf{P F}_{\mathbf{x}}=\mathbf{P} \frac{\partial \mathbf{F}}{\partial \mathbf{U}} \mathbf{U}_{\mathbf{x}}=\mathbf{A U}_{\mathbf{x}}
$$

where

$$
\mathbf{A}=\left(\begin{array}{cc}
a & -1 \\
-\nu / T_{r} & 0
\end{array}\right)
$$

Here, we have introduced $a=\partial f / \partial u$, which is not a constant but a function of $u$. Note that the preconditioned Jacobian is formally equivalent to the Jacobian of the linear time dependent hyperbolic advection-diffusion system. Hence, the eigenvalues and eigenvectors of this system are identical to those of the linear equations. It greatly simplifies the construction of the distribution matrices as described below. This is one of the advantages of the preconditioned conservative formulation.

To define the cell-residual, we first integrate the right hand side of Eq. (44) to get the unpreconditioned residual $\Psi^{C}$ :

$$
\begin{aligned}
\Psi^{C} & =\int_{x_{i}}^{x_{i+1}}\left(-\mathbf{F}_{x}+\mathbf{S}\right) d x \\
& =-\left(\mathbf{F}_{i+1}-\mathbf{F}_{i}\right)+\frac{\mathbf{S}_{i+1}+\mathbf{S}_{i}}{2}\left(x_{i+1}-x_{i}\right) .
\end{aligned}
$$

For the advective flux that is quadratic in the solution, it is well known that a conservative linearization exists:

$$
\Psi^{C}=-\frac{\overline{\partial \mathbf{F}}}{\partial \mathbf{U}}\left(\mathbf{U}_{i+1}-\mathbf{U}_{i}\right)+\frac{\mathbf{S}_{i+1}+\mathbf{S}_{i}}{2}\left(x_{i+1}-x_{i}\right)
$$

where $\frac{\overline{\partial \mathbf{F}}}{\partial \mathbf{U}}$ is the analytical Jacobian evaluated at the arithmetic average of the solution, $\left(U_{i+1}+U_{i}\right) / 2$ and it satisfies

$$
\mathbf{F}_{i+1}-\mathbf{F}_{i}=\frac{\overline{\partial \mathbf{F}}}{\partial \mathbf{U}}\left(\mathbf{U}_{i+1}-\mathbf{U}_{i}\right)
$$

exactly. The preconditioned cell-residual, which is to be distributed, is then given by

$$
\Phi^{C}=\overline{\mathbf{P}} \Psi^{C}
$$

where the matrix $\mathbf{P}$ is evaluated at the arithmetic average of the solution so that we have

$$
\Phi^{C}=-\overline{\mathbf{A}}\left(\mathbf{U}_{i+1}-\mathbf{U}_{i}\right)+\frac{\mathbf{S}_{i+1}+\mathbf{S}_{i}}{2}\left(x_{i+1}-x_{i}\right) .
$$


The distribution matrices can then be constructed in the same way as in the linear case based on $\overline{\mathbf{A}}$ :

$$
\begin{aligned}
& B_{C}^{-}=\frac{1}{\bar{a} L_{r}+2 \bar{\nu}}\left[\begin{array}{cc}
\bar{\nu} & \bar{\nu} L_{r} \\
\left(\bar{a} L_{r}+\bar{\nu}\right) / L_{r} & \bar{a} L_{r}+\bar{\nu}
\end{array}\right], \\
& B_{C}^{+}=\frac{1}{\bar{a} L_{r}+2 \bar{\nu}}\left[\begin{array}{cc}
\bar{a} L_{r}+\bar{\nu} & -\bar{\nu} L_{r} \\
-\left(\bar{a} L_{r}+\bar{\nu}\right) / L_{r} & \bar{\nu}
\end{array}\right] .
\end{aligned}
$$

where

$$
\bar{a}=\frac{a_{i}+a_{i+1}}{2}, \quad \bar{\nu}=\frac{\nu_{i}+\nu_{i+1}}{2} .
$$

The subscript $C$ indicates that these matrices are defined over the cell $C$. Note that the distribution matrices are not globally constant in general for nonlinear equations. The construction of the distribution matrices based on the conservative linearization is important for proper upwinding. If different averages are used, the distribution may not be strictly upwind. If the target equation does not allow the conservative linearization, the construction proposed in Ref. [21] may be employed to design a proper upwind distribution matrix. Note also that we evaluate $\nu$ in the source term by the average value over the cell to simplify the second component of the cell-residual as follows:

$$
\begin{gathered}
\Phi_{i}^{C}=\left[\begin{array}{c}
-\left(f_{i+1}-f_{i}\right)+\left(p_{i+1}-p_{i}\right)-\left(x_{i+1}-x_{i}\right) \frac{\alpha}{\Delta t}\left(u_{i+1}+u_{i}\right) / 2 \\
\frac{1}{\bar{T}_{r}}\left[\bar{\nu}\left(u_{i+1}-u_{i}\right)-\bar{p}\left(x_{i+1}-x_{i}\right)\right]
\end{array}\right]^{k+1} \\
+\left[\begin{array}{c}
\left(x_{i+1}-x_{i}\right)\left(s_{i+1}+s_{i}\right) / 2 \\
0
\end{array}\right]^{n-1, n},
\end{gathered}
$$

where

$$
\bar{T}_{r}=\frac{L_{r}}{\bar{a}+\bar{\nu} / L_{r}}
$$

Observe that we have $\bar{p}=\bar{\nu}\left(u_{i+1}-u_{i}\right) /\left(x_{i+1}-x_{i}\right)$ in the pseudo steady state. The cell-residual is then distributed to the nodes in all cells to yield the residual equation at node $i$ :

$$
0=\frac{1}{h_{i}}\left(B_{L}^{+} \Phi^{L}+B_{R}^{-} \Phi^{R}\right),
$$

where the pseudo-time derivatives have been dropped. 
We note that the Jacobian of this nonlinear advection-diffusion system is slightly different than that of the linear problem. The Jacobian blocks, however, are function of derivative of the distribution matrices $B^{-}$and $B^{+}$:

$$
\begin{aligned}
J_{i-1} & =\frac{1}{h_{i}} \frac{\partial\left(B_{L}^{+} \Phi^{L}\right)}{\partial U_{i-1}}, \\
J_{i} & =\frac{1}{h_{i}}\left(\frac{\partial\left(B_{L}^{+} \Phi^{L}\right)}{\partial U_{i}}+\frac{\partial\left(B_{R}^{-} \Phi^{R}\right)}{\partial U_{i}}\right), \\
J_{i+1} & =\frac{1}{h_{i}} \frac{\partial\left(B_{R}^{-} \Phi^{R}\right)}{\partial U_{i+1}} .
\end{aligned}
$$

In the special case $a / \nu=$ constant, which is the only case considered in this paper, the distribution matrices reduce entirely to constant matrices. Therefore, we can entirely ignore the derivatives of $B_{i}^{-}$and $B_{i}^{+}$in deriving the Jacobian. As a result, the Jacobians are given formally as in Eq. (26). This makes the implementation of both linear and nonlinear systems extremely easy and straightforward. Effects of nonlinearity arise only in the derivatives of the left and right cell-residuals:

$$
\begin{gathered}
\frac{\partial \Phi^{R}}{\partial U_{i}}=\left[\begin{array}{cc}
a_{i}-h_{R} \frac{\alpha}{2 \Delta t} & -1 \\
\frac{u_{i+1}-u_{i}}{\bar{T}_{r}} \frac{\partial \bar{\nu}}{\partial u_{i}}+\frac{\bar{\nu}\left(u_{i+1}-u_{i}\right)-\bar{p} h_{R}}{L_{r}}\left(\frac{\partial \bar{a}}{\partial u_{i}}+\frac{1}{L_{r}} \frac{\partial \bar{\nu}}{\partial u_{i}}\right)-\frac{\bar{\nu}}{\bar{T}_{r}} & -\frac{h_{R}}{2 \bar{T}_{r}}
\end{array}\right]^{k}, \\
\frac{\partial \Phi^{L}}{\partial U_{i}}=\left[\begin{array}{ccc}
(62) \\
-a_{i}-h_{L} \frac{\alpha}{2 \Delta t} & 1 \\
\frac{u_{i}-u_{i-1}}{\bar{T}_{r}} \frac{\partial \bar{\nu}}{\partial u_{i}}+\frac{\bar{\nu}\left(u_{i}-u_{i-1}\right)-\bar{p} h_{L}}{L_{r}}\left(\frac{\partial \bar{a}}{\partial u_{i}}+\frac{1}{L_{r}} \frac{\partial \bar{\nu}}{\partial u_{i}}\right)+\frac{\bar{\nu}_{r}}{\bar{T}_{r}} & -\frac{h_{L}}{2 \bar{T}_{r}}
\end{array}\right]^{k},
\end{gathered}
$$

where the average values indicated by the over bar are understood to be taken over the corresponding cell. Note that these derivatives depend on the solution at the current iteration, and therefore the superscript $k$ has been introduced. The same applies to the distribution matrices also. Obviously, the nonlinear system approaches the linear system in the event that both the advection and the diffusion coefficients are constant. Also, the boundary conditions can be implemented as described in Section 3.3 for the linear case. Therefore, our hyperbolic nonlinear time dependent advection-diffusion system scheme can be implemented for linear, nonlinear, steady and unsteady problems without loss of accuracy. For simplify the discussion we only showed Jacobian for nonlinear cases with $a / \nu=$ constant but the scheme works for general nonlinear problems. 


\section{Results}

In this section we present the results in three categories: 1) Steady advectiondiffusion equation for high Reynolds (or Peclet) number applications, 2) Unsteady linear advection-diffusion, and 3) Unsteady nonlinear advection-diffusion problems. We then present the order of accuracy results in the last subsection. Note that time-accurate computations are started by BDF1 in the first time step with extremely small time step (e.g. $t=10^{-8}$ ), and then by BDF2 thereafter with much larger time step (e.g. $t=0.001-0.5)$ for all unsteady cases. This will ensure that the order of accuracy stays second order through all times. We remark that explicit time stepping is not available for timeaccurate computations with the hyperbolic system method.

\subsection{Steady Linear Advection-Diffusion (High Re Appli- cations)}

Consider the advection-diffusion equation in $x \in(0,1)$ with $u(0)=0$ and $u(1)=1$ boundary conditions:

$$
\partial_{t} u+a \partial_{x} u=\nu \partial_{x x} u+s(x)
$$

where

$$
s(x)=\frac{\pi}{R e}[a \cos (\pi x)+\pi \nu \sin (\pi x)], \quad R e=a / \nu .
$$

This is a boundary layer problem with a non-trivial steady state solution in the diffusion limit as a result of the source term addition [2]. This equation develops a very narrow boundary layer near the right boundary $(x=1)$ where the advection becomes dominant. The exact steady state solution to this problem is given by

$$
u^{\text {exact }}(x)=\frac{e^{-R e}-e^{(x-1) R e}}{e^{-R e}-1}+\frac{1}{R e} \sin (\pi x) .
$$

We chose $R e$ values ranging from 1 to $10^{6}$ and solved the equation on nonuniform grid sizes up to 30000 nodes. We remark that the high-Re cases required extremely fine grids to meet the well-known requirement on the mesh Reynolds-number [2]. If desired, the computations can be performed on substantially coarser grids with more aggressive and customized grid stretching as well as with some nonlinear mechanism to prevent numerical oscillations. However, we simply refined the grid to meet the mesh Reynolds-number requirement because our method is powerful enough to solve the problem very efficiently (i.e., less than 10 Newton iterations) even on such dense grids. The ability to efficiently solve the problem on highly refined grids is a great advantage of these schemes. Shown in Fig. 5 are the comparisons between the 


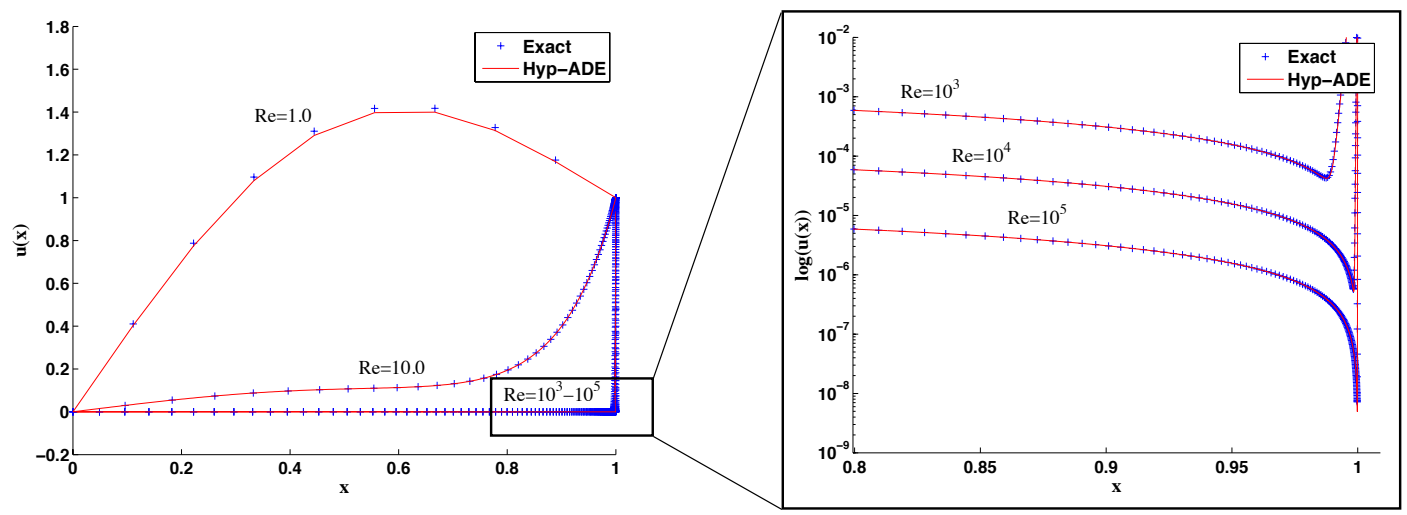

Figure 5: Residual-Distribution scheme on hyperbolic advection-diffusion system for boundary layer equation.

computational and the exact solutions. In this comparison, the exact analytic solution is represented with + (plus) symbols while the numerical results are shown with solid lines (denoted Hyp-ADE). The exact solutions are plotted at nodes, and therefore they also show the computational grids. The solutions were obtained with the Newton-GS method as described in Section 3.2. For this problems, within each Newton iteration, the GS relaxation were conducted until three orders of magnitude reduction is achieved in the linear solver. The computations were continued until the residuals of both the solution and the solution gradient were reduced by eight orders of magnitude. Table 1 gives the convergence data obtained using the implicit formulation of the hyperbolic advection-diffusion scheme. The linear dependency of the iterations on

Table 1: Boundary layer problem (Convergence criteria: Residuals $<10^{-8}$.)

\begin{tabular}{cccc}
\hline $\log _{10} R e$ & Number of nodes & GS relaxations/Newton iteration & Newton iteration \\
\hline 0 & 10 & 17 & 7 \\
0 & 100 & 324 & 7 \\
0 & 200 & 631 & 7 \\
0 & 300 & 967 & 7 \\
\hline 1 & 300 & 549 & 7 \\
2 & 300 & 131 & 7 \\
3 & 300 & 28 & 7 \\
4 & 300 & 38 & 6 \\
\hline 5 & 3000 & 60 & 6 \\
6 & 30000 & 50 & 5
\end{tabular}

the grid size is demonstrated at $R e=1$, which is a consequence of solving the advection-diffusion equation as a hyperbolic system for all Reynolds numbers 
through the diffusion limit. This is remarkable because the linear convergence is retained for any irregular grid in any dimensions ( $N$ is approximately the number of nodes in each coordinate direction in two and three dimensions) as demonstrated in Refs. [1-5]. In conventional methods, e.g., such as the alternating direction implicit method with Thomas' algorithm, the linear convergence can be achieved only on Cartesian grids (one-dimensional grid must exist in each coordinate direction). The $O(N)$ convergence on general problems and arbitrary grid systems makes the current scheme extremely attractive because it leads to orders of magnitude faster convergence in comparison with conventional methods whose convergence is typically $O\left(N^{2}\right)$.

Table 1 shows for the grid of 300 nodes that the number of GS relaxations decreases significantly as $R e$ increases. In Ref. [2], a formula for $L_{r}$ was derived that minimizes the condition number of the hyperbolic advection-diffusion system and a nearly uniform convergence was obtained for an explicit pseudotime marching scheme. We tested the formula in our method, but found that it did not change the significant decrease although it did reduce the number of GS relaxations approximately by $10 \%$. A different approach may be necessary to derive a formula to achieve $R e$-independent convergence of the GS relaxation in the implicit solver. Further study on $R e$-independent convergence is left as future work. Nevertheless, it should be noted that the number of Newton iterations is nearly $R e$-independent and very small: only 5 to 7 iterations to reduce the residual by eight orders of magnitude. Considering the fact that the cost of one GS relaxation is significantly cheaper than one Newton iteration, we find that the developed solver is still a very powerful steady solver. It thus serves as a very efficient solver for the unsteady residual equations as shown in the following sections.

Finally, we remark that the high- $R e$ cases required extremely fine grids to meet the well-known requirement on the mesh Reynolds-number as described in Ref. [2]. If desired, the computations can be performed on substantially coarser grids with more aggressive grid stretching. However, we simply refined the grid to meet the mesh Reynolds-number requirement because our method is powerful enough to solve the problem very efficiently (i.e., 5 to 7 Newton iterations) even on such dense grids. The ability to efficiently solve the problem on highly refined grids is a great advantage.

\subsection{Unsteady Linear Advection-Diffusion}

We present unsteady computations for the two types of boundary conditions; Dirichlet (or Neumann) and periodic. We again note that for hyperbolic system scheme the implementation of Dirichlet and Neumann boundary conditions are similar. 
Consider the time-dependent advection-diffusion equation in $x \in(0,1)$

$$
\partial_{t} u+a \partial_{x} u=\nu \partial_{x x} u
$$

with the following time-dependent boundary condition (Dirichlet):

$$
\begin{aligned}
& u(0, t)=0, \\
& u(1, t)=U \cos (\omega t),
\end{aligned}
$$

where $U$ is the amplitude of the oscillation and $\omega$ is the frequency of the oscillation on the right boundary. This problem has the following exact analytic solution:

$$
u^{\text {exact }}(x, t)=\operatorname{Real}\left(\frac{e^{\lambda_{1} x}-e^{\lambda_{2} x}}{e^{\lambda_{1}}-e^{\lambda_{2}}} U e^{i \omega t}\right), \lambda_{1,2}=\frac{a \pm \sqrt{a^{2}+4 i \omega \nu}}{2 \nu},
$$

where $i=\sqrt{-1}$. We solved this time-dependent advection-diffusion equation with the following first-order hyperbolic system:

$$
\begin{aligned}
\partial_{\tau} u+a \partial_{x} u & =\nu \partial_{x} p-\frac{3}{2 \Delta t} u+\frac{4 u^{n}-u^{n-1}}{2 \Delta t}, \\
\partial_{\tau} p & =\left(\partial_{x} u-p\right) / T_{r} .
\end{aligned}
$$

The solution evolution was started with the exact initial solution given as

$$
u(x, 0)=\operatorname{Real}\left(\frac{e^{\lambda_{1} x}-e^{\lambda_{2} x}}{e^{\lambda_{1}}-e^{\lambda_{2}}} U\right),
$$

and was advanced in physical time after the time-dependent residual was reduced by two orders of magnitude at each physical time step. We tested the scheme on a number of nonuniform grid systems ranging from $N=100 \ldots 10000$ nodes. Shown in Fig. 6 are the solution and the solution gradient obtained on the coarsest mesh $(\mathrm{N}=100)$ for $U=1, \nu=a=1$, and $\omega=7 \pi / 2$. We show the results on the coarsest mesh just to demonstrate the accuracy of the scheme. Similar results were obtained on finer grid systems. The results are then compared with the analytic solution. Here the numerical results (denoted Hyp-ADE) are over plotted with the exact solutions, meaning excellent agreement. We will show the residual values in the accuracy verification section.

We also obtained convergence data for different grid systems over 100 time steps. These are tabulated in Table 2, which shows a linear increase in number of iterations with increase of the grid system. This is, again, a consequence of solving the advection-diffusion equation as a hyperbolic system, where there are only first-derivatives and therefore the number of iterations is $O(N)$, not $O\left(N^{2}\right)$. It is also shown in Table 2 that only four Newton iterations are required for all grids considered. 

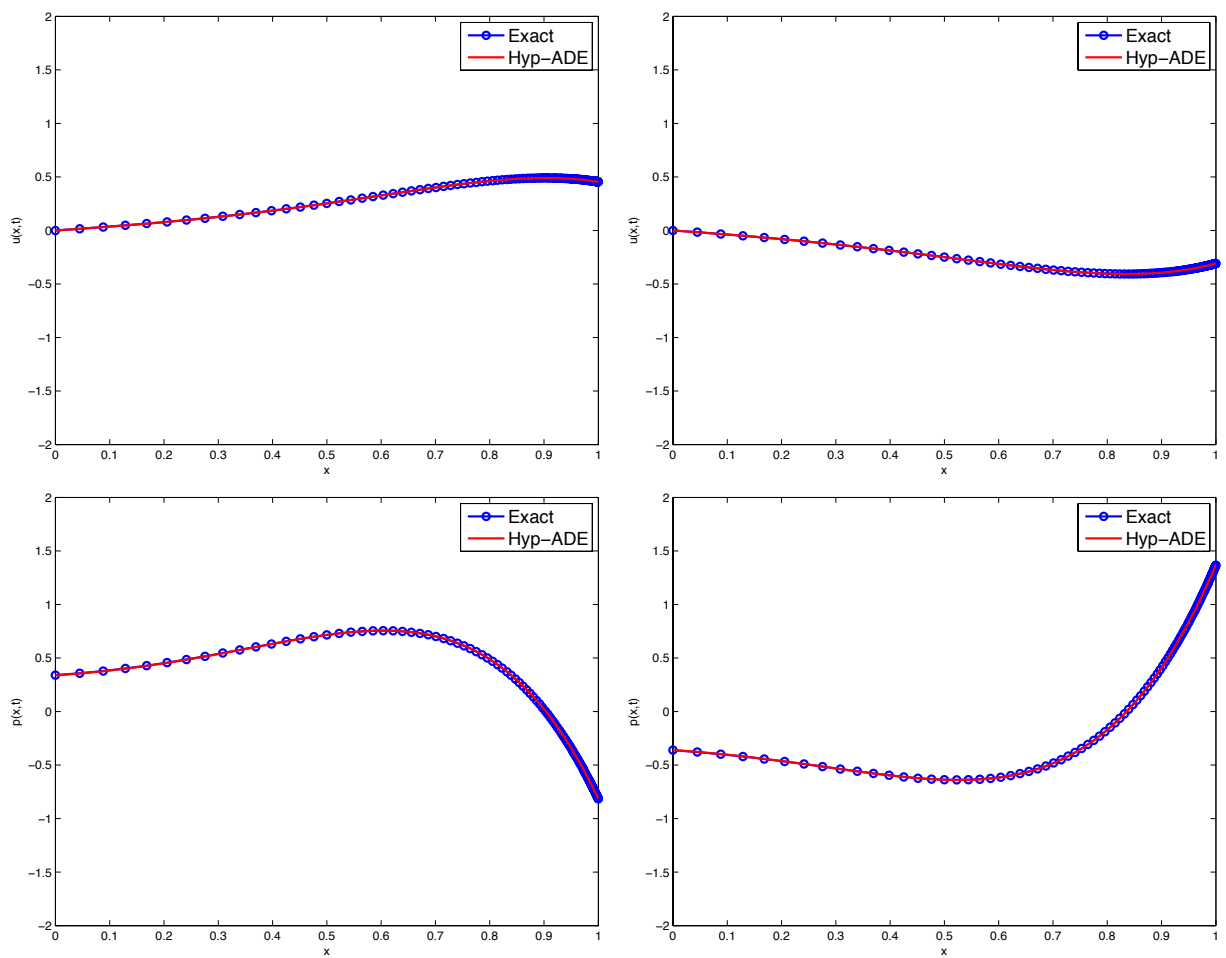

Figure 6: Time-dependent advection-diffusion with oscillatory boundary condition $u(0, t)=0, u(1, t)=\cos \left(\frac{7 \pi}{2} t\right)$ on $N=100$ irregular nodes. Time step $=$ $0.01 \mathrm{~s}$. Left figures at $t=0.1 \mathrm{~s}$, right figures at $t=0.4 \mathrm{~s}$.

Table 2: Hyperbolic time-dependent linear advection-diffusion problem with BDF2. Average data over 100 time steps are given. (Convergence criteria: Residuals $<10^{-2}$ )

\begin{tabular}{ccc}
\hline Number of nodes & GS relaxations/Newton iteration & Newton iterations \\
\hline 100 & 127 & 4 \\
300 & 370 & 4 \\
500 & 607 & 4 \\
1000 & 1201 & 4
\end{tabular}


For the periodic boundary condition demonstration, consider the timedependent advection-diffusion equation in $x \in(0,1)$ given as in Eq. (67) with the following initial condition:

$$
u(x, t=0)=\sin (\kappa x),
$$

where $\kappa$ is an arbitrary constant. The exact solution to this problem with a periodic boundary condition is:

$$
u^{\text {exact }}(x, t)=e^{-\kappa^{2} \nu t} \sin (\kappa(x-a t)) .
$$

We solved this problem with the same first-order hyperbolic advection-diffusions equation given as in Eqs. (71) and (72). For each physical time, we reduced the residuals by two orders of magnitude before advancing in time. During each time step, we also relaxed the linear system using GS relaxations until two orders of magnitude reduction in the linear system residuals was achieved. Shown in Fig. 7 are the solution and solution gradient on $N=25$ uniform grid
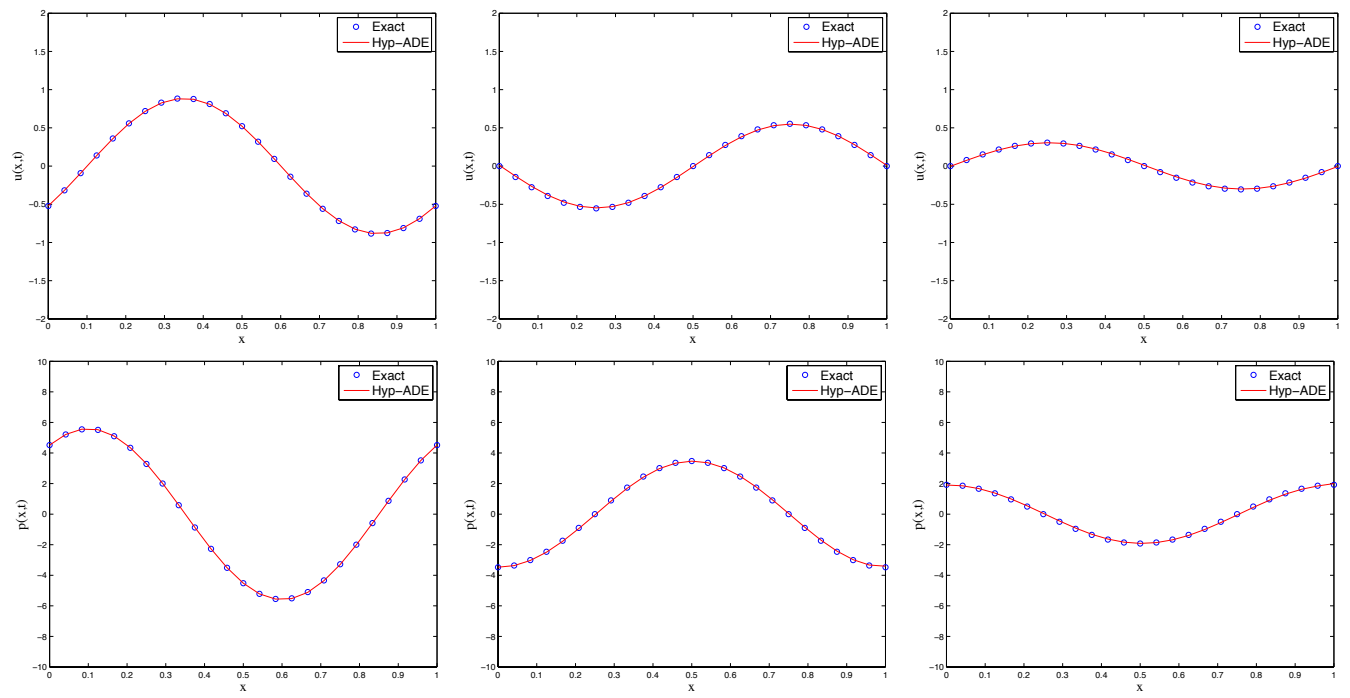

Figure 7: Time-dependent linear advection-diffusion problem $(a=1, \nu=0.03)$ with periodic boundary condition on $N=25$ uniform nodes. Time step $=$ $0.01 \mathrm{~s}$. From left to right, solutions at $t=0.1 \mathrm{~s}, 0.5 \mathrm{~s}, 1.0 \mathrm{~s}$.

for $a=1, \nu=0.03$, and $\kappa=2 \pi$. The results (Hyp-ADE) are over plotted with the exact solution, indicating excellent accuracy on such a coarse grid. Similar results were also obtained for larger grid systems.

We examined the convergence rate of this problem on several grid systems. Given in Table 3, are the average numbers of GS relaxations per Newton iteration obtained over 100 time steps. Clearly the convergence rate is of $O(N)$, not $O\left(N^{2}\right)$ as typical for numerical methods for the advection-diffusion equation. Observe that for most grid systems only one or two Newton iterations were 
Table 3: Hyperbolic time-dependent linear advection-diffusion problem with periodic $\mathrm{BC}(a=1, \nu=0.03)$ Average data over 100 time steps are given.(Convergence criteria: Residuals $<10^{-2}$ )

\begin{tabular}{ccc}
\hline Number of nodes & GS relaxations/Newton iteration & Newton iterations \\
\hline 25 & 4 & 3 \\
100 & 11 & 3 \\
300 & 33 & 2 \\
500 & 55 & 2 \\
1000 & 116 & 2
\end{tabular}

sufficient to obtain accurate solutions. We also note that we used $\Delta t=0.01-$ sec for all the grid systems; the BDF schemes are unconditionally stable. The time step is orders-of-magnitude larger than that required for conventional explicit schemes, which is limited by $O\left(h^{2}\right)$. Of course, conventional implicit schemes also allow unconditionally large time steps, but it requires $O\left(N^{2}\right)$ convergence in an iterative linear solver and potentially a much larger number of outer iterations as well if the exact linearization is not possible and Newton's method cannot be constructed. Hence, the method developed here has two major advantages over conventional methods: the exact linearization (Newton's method) and $O(N)$ iterative convergence in the linear solver. The latter advantage can be potentially huge with increase of the grid system as the speed-up factor is $O(N)$ and thus grows for finer grids. For example, for $N=1000$, our scheme is at least four orders of magnitude faster than the conventional schemes, which is remarkable.

\subsection{Unsteady Nonlinear Advection-Diffusion}

Consider the time-dependent nonlinear viscous Burgers equation with a timedependent source term:

$$
\partial_{t} u+\partial_{x} f=\partial_{x}\left(\nu u_{x}\right)+g(x, t), \quad x \in(0,1),
$$

where $f=u^{2} / 2, \nu=u$, and

$$
g(x, t)=u_{t}^{e}+\frac{1}{2}\left(\left(u^{e}\right)^{2}\right)_{x}-\left(u_{x}^{e}\right)^{2}-u^{e} u_{x x}^{e} .
$$

The source term has been generated by the following function:

$$
u^{e}(x, t)=\operatorname{Real}\left(\frac{\sinh (x \sqrt{i \omega / \nu})}{\sinh (\sqrt{i \omega / \nu})} U e^{i \omega t}\right)+C, C>1,
$$


so that it is the exact solution to Eq. (76). The boundary conditions are

$$
\begin{aligned}
& u(0, t)=C, \\
& u(1, t)=C+U \cos (\omega t),
\end{aligned}
$$

where $\omega$ is the frequency of the oscillation on the right boundary, and $U$ is the amplitude of the oscillation. The constant $C$ must be greater than 1 to keep the diffusion coefficient positive.

We solved this time-dependent nonlinear advection-diffusion equation with the following equivalent first-order hyperbolic system:

$$
\begin{aligned}
\partial_{\tau} u+\partial_{x}\left(u^{2}\right) & =\partial_{x} p-\frac{3}{2 \Delta t} u+\frac{4 u^{n}-u^{n-1}}{2 \Delta t}+g(x, t), \\
\frac{T_{r}}{\nu} \partial_{\tau} p & =\left(\partial_{x} u-p / \nu\right) .
\end{aligned}
$$

Shown in Fig. 8 are the results obtained on $N=100$ nonuniform nodes for $U=1, \omega=7 \pi / 2$, and $C=2$. The scheme produces very accurate time
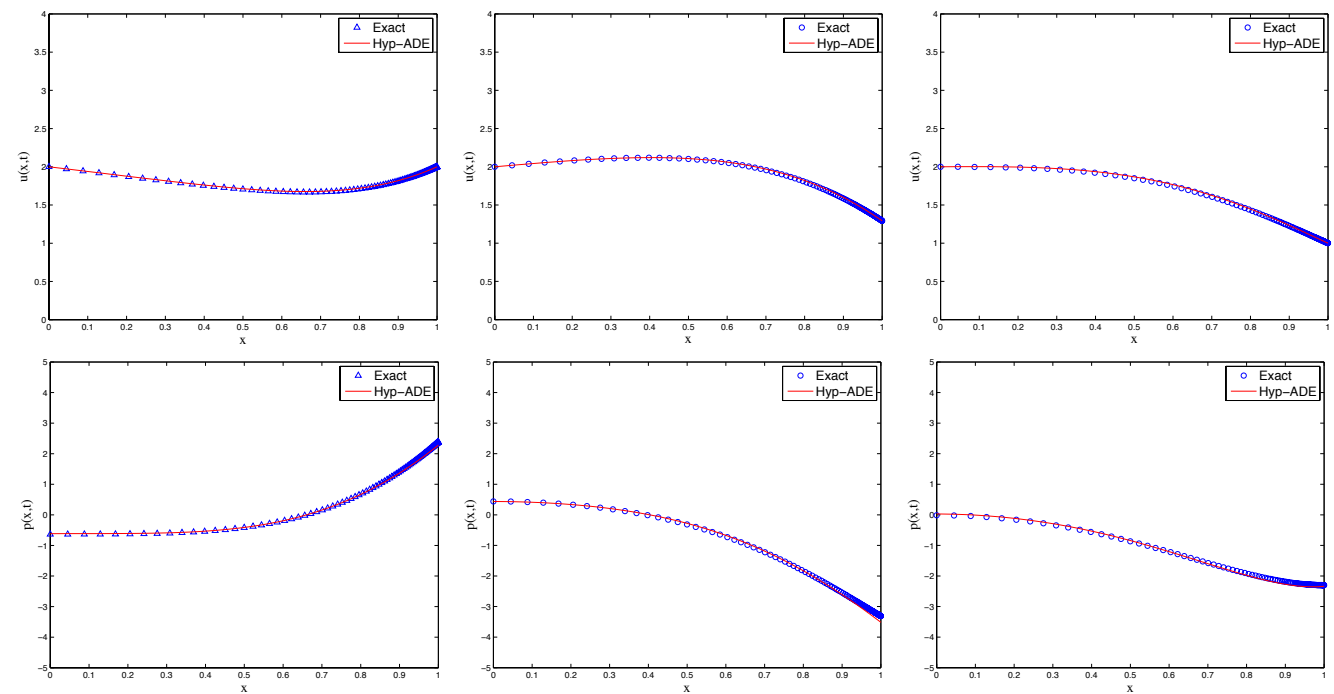

Figure 8: Time-dependent nonlinear advection-diffusion $(a=\nu=u)$ with oscillatory boundary condition $u(0, t)=2, u(1, t)=2+\cos \left(\frac{7 \pi}{2} t\right)$ on $N=100$ nonuniform nodes. Time step $=0.01 \mathrm{~s}$. From left to right, solutions at $t=$ $0.1 s, 2.5 s, 10.0 s$.

evolutions of the solution $(u)$ and the gradient $(p / \nu)$ on nonuniform grids (see also Fig. 9.)

The $O(N)$ convergence rate was once again achieved for the time-dependent nonlinear hyperbolic advection-diffusion system. This is given in Table 4, where the average number of iterations were obtained over 1000 time steps 

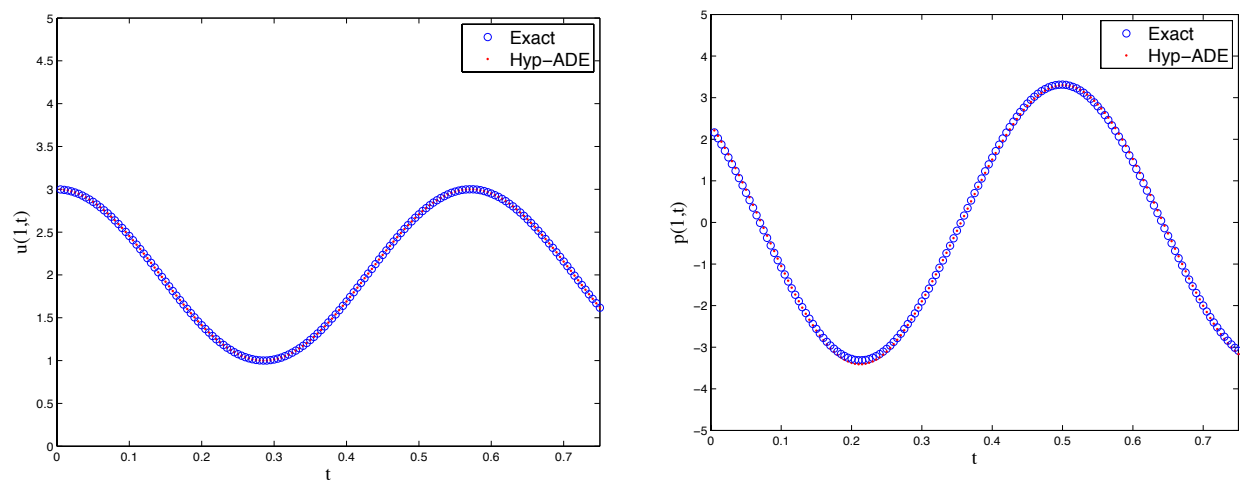

Figure 9: Time-dependent nonlinear advection-diffusion $(a=\nu=u)$ with oscillatory boundary condition $u(0, t)=2, u(1, t)=2+\cos \left(\frac{7 \pi}{2} t\right)$ on $N=100$ nonuniform nodes. Time step $=0.01 \mathrm{~s}$

(over 17 periods). Note also that the method converged at four Newton iterations for all grids considered. Again, a constant time step ( $\Delta t=0.01-\mathrm{sec})$ was used for all the grid systems. And the method is substantially more efficient here also than conventional explicit/implicit schemes as discussed for the linear case in Section 6.2.

Table 4: Hyperbolic time-dependent nonlinear advection-diffusion problem with BDF2. Average data over 100 time steps are given.(Convergence criteria: Residuals $<10^{-2}$ )

\begin{tabular}{ccc}
\hline Number of nodes & GS relaxations/Newton iteration & Newton iterations \\
\hline 100 & 88 & 4 \\
300 & 263 & 4 \\
500 & 440 & 4 \\
1000 & 882 & 4
\end{tabular}

\subsection{Accuracy Verification}

To demonstrate the overall order of accuracy of our time-dependent hyperbolic advection-diffusion scheme, we computed the $L_{1}=\sum_{i=1}^{N}\left(U_{i}^{\text {exact }}-U_{i}\right) / N$ as well as $L_{\infty}=\operatorname{Max}\left(U_{i}^{\text {exact }}-U_{i}\right)$ norms of errors on a series of nonuniform grids of $N$ nodes, and time step increments $\Delta t$. For each case, we terminate the solver when the residuals were dropped by two orders of magnitude. We remark that in some practical applications two orders of magnitude reduction in the residuals may not be sufficient to achieve the appropriate order of accuracy. But for the cases considered here, terminating at even nine orders of magnitude 
resulted in similar order of accuracy. The spatial accuracy was obtained by comparing several nonuniform grid systems on a fixed $\Delta t$, while the temporal accuracy was obtained by comparing various $\Delta t$ on a fixed nonuniform grid system. We also included boundary nodes in both $L_{1}$ and $L_{\infty}$ norms. For unsteady cases, the $L_{1}$ and $L_{\infty}$ norms were evaluated at $t=1.0-$-sec.
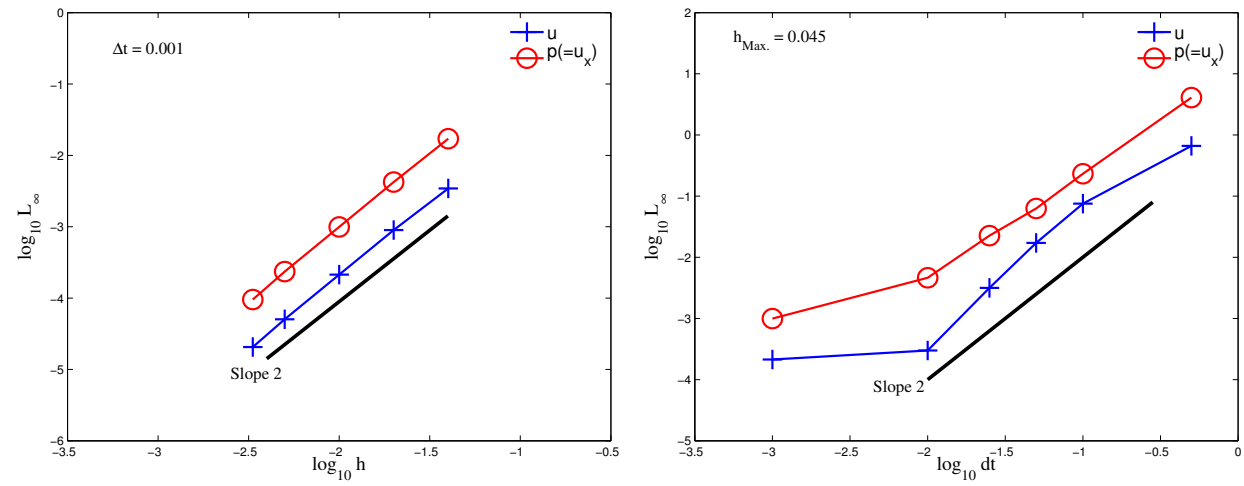

Figure 10: $L_{\infty}$ error obtained for time-dependent linear hyperbolic advectiondiffusion system scheme. Left: Spatial accuracy, Right: Temporal accuracy.

Figures 10 and 11 show the $L_{\infty}$ error convergence results for both linear and nonlinear time-dependent hyperbolic advection diffusion system, where $h$ is the representative mesh spacing defined by $h=/(N-1)$. For discussion purposes, we only present the accuracy plots for $R e=1$ results; similar results were obtained for other Reynolds numbers. These results show that our scheme is second-order accurate for all the variables and the gradients (note that again that our flux integral is exact) at all the grid nodes including the boundary nodes (see also Tables 5, 6, 7, and 8). We note that it is natural for the error convergence in the temporal space to deteriorate when the $O\left(\Delta t^{2}\right)<<O\left(h^{2}\right)$ as well as when $\Delta t$ is too large for the time discretization to be in asymptotic range; i.e. a lower order of accuracy for coarse discretization in time is typical. These limits are also included in the temporal accuracy plot for completeness.

Table 5: Spatial accuracy for the linear advection-diffusion problem with oscillatory boundary condition on several nonuniform grid systems $(\Delta t=0.001 s)$.

\begin{tabular}{ccccccc}
\hline Number of nodes & $L_{1}$ error of $u$ & $L_{\infty}$ error of $u$ & Order & $L_{1}$ error of $p$ & $L_{\infty}$ error of $p$ & Order \\
\hline 25 & $9.65 \mathrm{E}-04$ & $3.44 \mathrm{E}-03$ & & $6.58 \mathrm{E}-03$ & $1.71 \mathrm{E}-02$ & \\
50 & $2.28 \mathrm{E}-05$ & $8.98 \mathrm{E}-04$ & 1.94 & $1.08 \mathrm{E}-03$ & $4.22 \mathrm{E}-03$ & 2.02 \\
100 & $6.10 \mathrm{E}-05$ & $2.13 \mathrm{E}-04$ & 2.08 & $3.08 \mathrm{E}-04$ & $9.95 \mathrm{E}-04$ & 2.08 \\
200 & $1.59 \mathrm{E}-06$ & $5.04 \mathrm{E}-05$ & 2.08 & $8.76 \mathrm{E}-05$ & $2.35 \mathrm{E}-04$ & 2.08 \\
300 & $7.58 \mathrm{E}-06$ & $2.06 \mathrm{E}-05$ & 2.21 & $4.87 \mathrm{E}-05$ & $9.53 \mathrm{E}-05$ & 2.23
\end{tabular}



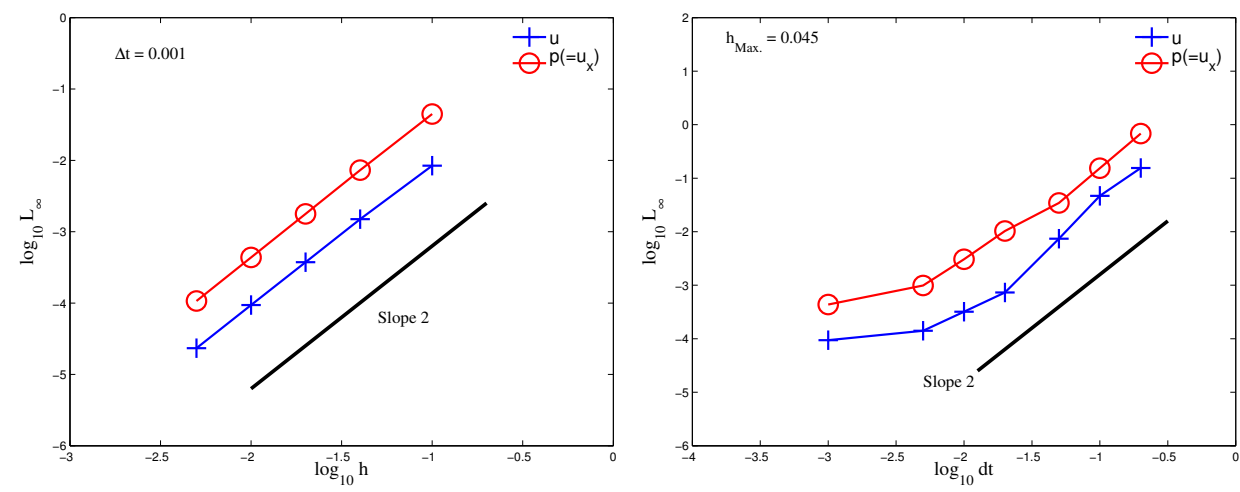

Figure 11: $L_{\infty}$ error obtained for time-dependent nonlinear hyperbolic advection-diffusion system scheme. Left: Spatial accuracy, Right: Temporal accuracy.

Table 6: Temporal accuracy for the linear advection-diffusion problem with oscillatory boundary condition on a nonuniform grid $(\mathrm{N}=100)$ with $h_{\text {Max. }}=$ 0.045 .

\begin{tabular}{ccccccc}
\hline$\Delta t$ & $L_{1}$ error of $u$ & $L_{\infty}$ error of $u$ & Order & $L_{1}$ error of $p$ & $L_{\infty}$ error of $p$ & Order \\
\hline 0.500 & $3.01 \mathrm{E}-01$ & $6.63 \mathrm{E}-01$ & & $2.46 \mathrm{E}+00$ & $4.09 \mathrm{E}+00$ & \\
0.100 & $2.32 \mathrm{E}-02$ & $7.55 \mathrm{E}-02$ & 1.35 & $1.25 \mathrm{E}-01$ & $2.32 \mathrm{E}-01$ & 1.78 \\
0.050 & $4.37 \mathrm{E}-03$ & $1.72 \mathrm{E}-02$ & 2.13 & $3.57 \mathrm{E}-02$ & $6.28 \mathrm{E}-02$ & 1.88 \\
0.025 & $9.66 \mathrm{E}-04$ & $3.16 \mathrm{E}-03$ & 2.44 & $1.16 \mathrm{E}-02$ & $2.26 \mathrm{E}-02$ & 1.47 \\
0.010 & $1.48 \mathrm{E}-04$ & $2.99 \mathrm{E}-04$ & 2.57 & $2.21 \mathrm{E}-03$ & $4.63 \mathrm{E}-03$ & 1.73 \\
0.001 & $6.02 \mathrm{E}-05$ & $2.13 \mathrm{E}-04$ & 0.15 & $3.05 \mathrm{E}-04$ & $9.95 \mathrm{E}-04$ & 0.67
\end{tabular}

Table 7: Spatial accuracy for the nonlinear advection-diffusion problem with oscillatory boundary condition on several nonuniform grid systems $(\Delta t=$ $0.001 s)$.

\begin{tabular}{ccccccc}
\hline Number of nodes & $L_{1}$ error of $u$ & $L_{\infty}$ error of $u$ & Order & $L_{1}$ error of $p$ & $L_{\infty}$ error of $p$ & Order \\
\hline 10 & $1.83 \mathrm{E}-03$ & $8.41 \mathrm{E}-03$ & & $1.20 \mathrm{E}-02$ & $4.46 \mathrm{E}-02$ & \\
25 & $4.36 \mathrm{E}-04$ & $1.50 \mathrm{E}-03$ & 1.88 & $2.79 \mathrm{E}-03$ & $7.29 \mathrm{E}-03$ & 1.98 \\
50 & $9.00 \mathrm{E}-05$ & $3.74 \mathrm{E}-04$ & 2.00 & $5.48 \mathrm{E}-04$ & $1.78 \mathrm{E}-03$ & 2.03 \\
100 & $2.80 \mathrm{E}-05$ & $9.39 \mathrm{E}-05$ & 1.99 & $1.41 \mathrm{E}-04$ & $4.36 \mathrm{E}-04$ & 2.03 \\
200 & $8.19 \mathrm{E}-06$ & $2.33 \mathrm{E}-05$ & 2.01 & $4.81 \mathrm{E}-05$ & $1.07 \mathrm{E}-04$ & 2.03
\end{tabular}


Table 8: Temporal accuracy for the nonlinear advection-diffusion problem with oscillatory boundary condition on a nonuniform grid system $(\mathrm{N}=100)$ with $h_{\text {Max. }}=0.045$.

\begin{tabular}{ccccccc}
\hline$\Delta t$ & $L_{1}$ error of $u$ & $L_{\infty}$ error of $u$ & Order & $L_{1}$ error of $p$ & $L_{\infty}$ error of $p$ & Order \\
\hline 0.200 & $6.46 \mathrm{E}-02$ & $1.55 \mathrm{E}-01$ & & $4.97 \mathrm{E}-01$ & $6.83 \mathrm{E}-01$ & \\
0.100 & $1.52 \mathrm{E}-02$ & $4.67 \mathrm{E}-02$ & 1.73 & $9.00 \mathrm{E}-02$ & $1.54 \mathrm{E}-01$ & 2.15 \\
0.050 & $2.03 \mathrm{E}-03$ & $7.40 \mathrm{E}-03$ & 2.66 & $1.99 \mathrm{E}-02$ & $3.45 \mathrm{E}-02$ & 2.16 \\
0.020 & $3.52 \mathrm{E}-04$ & $7.32 \mathrm{E}-04$ & 2.52 & $5.01 \mathrm{E}-03$ & $1.03 \mathrm{E}-02$ & 1.32 \\
0.010 & $1.34 \mathrm{E}-04$ & $3.20 \mathrm{E}-04$ & 1.19 & $1.70 \mathrm{E}-03$ & $3.06 \mathrm{E}-03$ & 1.75 \\
0.005 & $6.68 \mathrm{E}-05$ & $1.41 \mathrm{E}-04$ & 1.18 & $6.20 \mathrm{E}-04$ & $9.90 \mathrm{E}-04$ & 1.63 \\
0.001 & $2.80 \mathrm{E}-05$ & $9.39 \mathrm{E}-05$ & 0.25 & $1.41 \mathrm{E}-04$ & $4.36 \mathrm{E}-04$ & 0.51
\end{tabular}

\section{Conclusions}

In this paper, we have extended the first-order hyperbolic system method to time-accurate computations. The time integration scheme is implicit with the second-order backward-difference formula, and the resulting system of timedependent residual equations is solved by a steady solver developed based on the hyperbolic method. The steady solver is Newton's method constructed from a compact upwind residual-distribution scheme for linear and nonlinear advection-diffusion equations. The construction of the numerical scheme was greatly simplified for the nonlinear equation by the preconditioned conservative formulation. We have demonstrated that the developed time-accurate schemes are capable, at every physical time step, of producing second-order accurate solution and gradient on highly refined nonuniform grids by less than five Newton iterations with the linear convergence of the block GS relaxations for all test cases considered. Thus, the notable features of the hyperbolic method have been shown to carry over to time-dependent problems.

The developed methodology based on the second-order backward Euler time integration can be extended to practical one-dimensional problems [7-11], bringing significant improvements in both accuracy and efficiency. Extension to higher-order accuracy is also possible by improving the residual evaluation and higher-order backward-difference formulas. It is relatively simple in one dimension because the integration of the flux balance term is exact in one dimension as mentioned in Section 3.1. In higher dimensions, although the construction of time-accurate schemes is as simple as presented in the paper, it is known that a high-order quadrature would be required for the flux balance integration in order to achieve the equal order of spatial accuracy in the solution and the gradient [2]. Although not considered in the present paper, the construction of non-oscillatory schemes is an important area of development for problems with discontinuities such as shock waves. 


\section{Acknowledgments}

The second author acknowledges the support by the U.S. Army Research Office under the contract/grant number W911NF-12-1-0154 with Dr. Frederick Ferguson as the program manager.

\section{References}

1. H. Nishikawa. A first-order system approach for diffusion equation. I: Second-order residual-distribution schemes. J. Comput. Phys., 227:315$352,2007$.

2. H. Nishikawa. A first-order system approach for diffusion equation. II: Unification of advection and diffusion. J. Comput. Phys., 229:3989-4016, 2010 .

3. H. Nishikawa. New-generation hyperbolic Navier-Stokes schemes: $O(1 / h)$ speed-up and accurate viscous/heat fluxes. In Proc. of 20th AIAA Computational Fluid Dynamics Conference, AIAA Paper 2011-3043, Honolulu, Hawaii, 2011.

4. H. Nishikawa. First, second, and third order finite-volume schemes for diffusion. In Proc. of 51st AIAA Aerospace Sciences Meeting, AIAA Paper 2011-1125, Grapevine, Texas, January 2013.

5. H. Nishikawa. First, second, and third order finite-volume schemes for advection-diffusion. In Proc. of 21st AIAA Computational Fluid Dynamics Conference, AIAA Paper 2011-2568, San Diego, California, June 2013.

6. A. Jameson. Time dependent calculations using multigrid, with applications to unsteady flows past airfoils and wings. AIAA Paper 91-1596, 1991.

7. Y.-K. Chen and F. S. Milos. Ablation and thermal response program for spacecraft heatshield analysis. J. Spacecraft and Rockets, 36:475-583, 1999.

8. M. Mahzari, R. D. Braun, T. White, and D. Bose. Preliminary analysis of the mars science laboratory entry aerothermodynamic environment and thermal protection system performance. In Proc. of 51st AIAA Aerospace Sciences Meeting including the New Horizons Forum and Aerospace, AIAA Paper 2013-0185, Grapevine, Texas, January 2013.

9. D. Bose, T. White, M. Mahzari, and K. Edquist. A reconstruction of aerothermal environment and thermal protection system response of the 
mars science laboratory entry vehicle. In Proc. of American Astronomical Society 221st Meeting, AAS 2013-311-0712, Long Beach, California, January 2013.

10. N. R. Merski. Reduction and analysis of phosphor thermography data with the IHEAT software package. In Proc. of 36th AIAA Aerospace Sciences Meeting and Exhibits, AIAA Paper 98-0712, Reno, Nevada, January 1998.

11. T. J. Horvath and s. A. Berry and b. R. Hollis and d. S. Liechty and h. H. Hamilton and n. R. Merski. J. Spacecraft and Rockets, 38:635-645, 2001.

12. A. Ferrante and H. Deconinck. Solution of the unsteady euler equations using residual distribution and flux corrected transport. In Technical Report VKI-PR 97-08. 1997.

13. A. Csik, M. Ricchiuto, H. Deconninck, and S. Poedts. Space-time residual distribution schemes for hyperbolic conservation laws. In Proc. of 15th AIAA Computational Fluid Dynamics Conference, AIAA Paper 20012617, Anaheim, 2001.

14. R. Abgrall and M. Mezine. Construction of second order accurate monotone and stable residual distribution schemes for unsteady flow problems. J. Comput. Phys., 188:16-55, 2002.

15. A. Bonfiglioli and R. Paciorri. A mass-matrix formulation of unsteady fluctuation splitting schemes consistent with Roe's parameter vector. Int. J. Comput. Fluid Dyn., 27, 2013.

16. G. Rossiello, P. De Palma, G. Pascazio, and M. Napolitano. Third-orderaccurate fluctuation-splitting schemes for unsteady hyperbolic problems. J. Comput. Phys., 222:332-352, 2007.

17. D. Caraeni and L. Fuchs. Compact third-order multidimensional upwind scheme for Navier-Stokes simulations. Appl. Math., 15:373-401, 2002.

18. M. Ricchiuto and R. Abgrall. Explicit Runge-Kutta residual distribution schemes for time dependent problems: Second order case. J. Comput. Phys., 229:5653-5691, 2010.

19. M. Ricchiuto and H. Nishikawa. Dual-time hyperbolic diffusion systems. Unpublished, 2012.

20. U. Trottenberg, C. W. Oosterlee, and A. Schüller. Multigrid. Academic Press, 2000.

21. A. Csik, M. Ricchiuto, and H. Deconinck. A conservative formulation of the multidimensional upwind residual distribution schemes for general nonlinear conservation laws. J. Comput. Phys., 179:286-312, 2002. 


\begin{tabular}{|c|c|c|c|c|c|}
\hline \multicolumn{5}{|c|}{ REPORT DOCUMENTATION PAGE } & $\begin{array}{l}\text { Form Approved } \\
\text { OMB No. } 0704-0188\end{array}$ \\
\hline \multicolumn{6}{|c|}{ 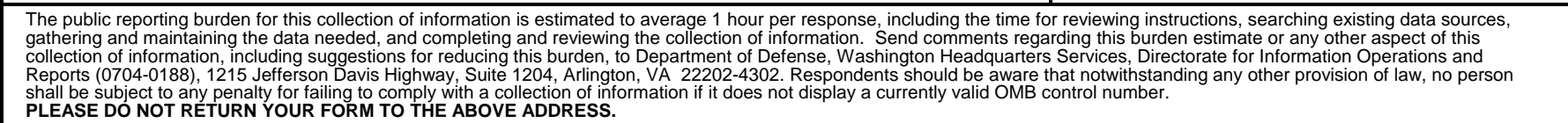 } \\
\hline \multicolumn{5}{|c|}{\begin{tabular}{|c|l|} 
1. REPORT DATE (DD-MM-YYYY) & $\begin{array}{l}\text { 2. REPORT TYPE } \\
01-03-2014\end{array}$ \\
Technical Memorandum
\end{tabular}} & 3. DATES COVERED (From - To) \\
\hline \multirow{3}{*}{\multicolumn{5}{|c|}{$\begin{array}{l}\text { 4. TITLE AND SUBTITLE } \\
\text { First-Order Hyperbolic System Method for Time-Dependent } \\
\text { Advection-Diffusion Problems }\end{array}$}} & 5a. CONTRACT NUMBER \\
\hline & & & & & 5b. GRANT NUMBER \\
\hline & & & & & 5c. PROGRAM ELEMENT NUMBER \\
\hline \multicolumn{5}{|c|}{ 6. AUTHOR(S) } & 5d. PROJECT NUMBER \\
\hline \multirow{3}{*}{\multicolumn{5}{|c|}{ Mazaheri, Alireza; Nishikawa, Hiroaki }} & 5e. TASK NUMBER \\
\hline & & & & & 5f. WORK UNIT NUMBER \\
\hline & & & & & 747797.02.09.30.04 \\
\hline \multicolumn{5}{|c|}{$\begin{array}{l}\text { 7. PERFORMING ORGANIZATION NAME(S) AND ADDRESS(ES) } \\
\text { NASA Langley Research Center } \\
\text { Hampton, VA } 23681-2199\end{array}$} & $\begin{array}{l}\text { 8. PERFORMING ORGANIZATION } \\
\text { REPORT NUMBER } \\
\text { L-20378 }\end{array}$ \\
\hline \multirow{3}{*}{\multicolumn{5}{|c|}{$\begin{array}{l}\text { 9. SPONSORING/MONITORING AGENCY NAME(S) AND ADDRES } \\
\text { National Aeronautics and Space Administration } \\
\text { Washington, DC 20546-0001 }\end{array}$}} & 10. SPONSOR/MONITOR'S ACRONYM(S) \\
\hline & & & & & NASA \\
\hline & & & & & $\begin{array}{l}\text { 11. SPONSOR/MONITOR'S REPORT } \\
\text { NUMBER(S) } \\
\text { NASA/TM-2014-218175 }\end{array}$ \\
\hline \multicolumn{6}{|c|}{$\begin{array}{l}\text { 12. DISTRIBUTION/AVAILABILITY STATEMENT } \\
\text { Unclassified - Unlimited } \\
\text { Subject Category } 64 \\
\text { Availability: NASA CASI (443) } 757-5802\end{array}$} \\
\hline \multicolumn{6}{|c|}{ 13. SUPPLEMENTARY NOTES } \\
\hline \multicolumn{6}{|c|}{$\begin{array}{l}\text { 14. ABSTRACT } \\
\text { A time-dependent extension of the first-order hyperbolic system method for advection-diffusion problems is introduced. } \\
\text { Diffusive/viscous terms are written and discretized as a hyperbolic system, which recovers the original equation in the steady } \\
\text { state. The resulting scheme offers advantages over traditional schemes: a dramatic simplification in the discretization, } \\
\text { high-order accuracy in the solution gradients, and orders-of-magnitude convergence acceleration. The hyperbolic } \\
\text { advection-diffusion system is discretized by the second-order upwind residual-distribution scheme in a unified manner, and the } \\
\text { system of implicit-residual-equations is solved by Newton's method over every physical time step. The numerical results are } \\
\text { presented for linear and nonlinear advection-diffusion problems, demonstrating solutions and gradients produced to the same } \\
\text { order of accuracy, with rapid convergence over each physical time step, typically less than five Newton iterations. }\end{array}$} \\
\hline \multicolumn{6}{|c|}{ 15. SUBJECT TERMS } \\
\hline \multicolumn{6}{|c|}{ Finite Volume; Higher-Order Scheme; Nonlinear; Residual Distribution; Unsteady } \\
\hline \multicolumn{3}{|c|}{ 16. SECURITY CLASSIFICATION OF: } & 17. LIMITATION OF & 18. NUMBER & 19a. NAME OF RESPONSIBLE PERSON \\
\hline a. REPORT & \multicolumn{2}{|c|}{ b. ABSTRACT/C. THIS PAGE } & & PAGES & STI Help Desk (email: help@sti.nasa.gov) \\
\hline $\mathrm{U}$ & $\mathrm{U}$ & $\mathrm{U}$ & & & (443) $757-5802$ \\
\hline
\end{tabular}

